\title{
Effectiveness of Online Reporting in A Laboratory of A Tertiary Care Hospital - A Preliminary Study
}

\author{
Suresh Babu Ganji ${ }^{1}$, Pavani Kiranmai Thandu ${ }^{2}$, Suneetha Revupalli*, Anitha \\ Ramtenki ${ }^{3}$, Sudhakar Kavali*, Sameena Rahman, Ajitha Ganga, Hemalatha \\ Desam, Laxmi Katna, Anil Kumar Malkajgiri, Rajeshwari Bommeraboyina, \\ Madhukar Madapa, Umakanth Kheni \\ ${ }^{1}$ Asst. Professor, Lab I/c \& corresponding author: sushwasa@gmail.com \\ ${ }^{2}$ Senior Resident; 3.PG II Year;* Biochemist; All Others Are Lab Technical Staff Participated In This Study.
}

\begin{abstract}
:
Objective: Errors in laboratory can occur at all levels; with the advent of technology errors in analytical stage were decreased compared to pre \& post analytical stages. Advances in software technology and its integration in healthcare industry as HIS and LIS is a step forward to minimize pre and post analytical errors in the laboratory. In this study the effectiveness of online software in reporting results is compared with older manual reporting.

Methods: Our one year old Health 4all (YouSee) online software with non interfaced instrumentation at Biochemistry laboratory was reviewed for its efficacy. We took feedback by Laboratory staff, clinical staff and patient attenders by interviews and opinions noted for drawing conclusions.

Discussion: This study highlighted the importance of selection of proper and suitable software for a particular hospital, need of appropriate staff training and how it adds benefit in improving the quality of results and safety of patient data.

Conclusion: Importance of integration of administration and laboratories in understanding the challenges; and acting in right direction by retrospection, can deliver quicker and better performance thus improving the quality of services and patient care.
\end{abstract}

Keywords: HIS hospital information system, LIS laboratory information system, COMPUTERS, Online Reporting, Pre analytical \& Post Analytical Errors.

\section{Introduction}

Errors in laboratory medicine are intrinsically obscure as they are difficult to identify and, when found, are less easily understood than other types of medical error. Medical errors can no longer be seen as inevitable, but as something that can be actively streamlined and prevented (1).Compared with adverse events related to surgery or other treatment errors that are often glaring and obvious, laboratory errors tend to be more insidious and difficult to pinpoint in time and place (2). Physicians responsible for making clinical decisions seldom do not perceive laboratory errors as a harmful source of patient adverse events, nor do they understand that most laboratory defects may arise from the pre- and post-analytic steps. Some of these "llaboratory sentinel events" have already been identified, including inappropriate test requests and patient misidentification (pre-analytical phase), use of wrong assays, severe analytical errors, tests performed on unsuitable samples, release of lab results in spite of poor quality controls (analytical phase), and failure to alert critical values and wrong report destination (post-analytical phase) $(3,4)$. Modern robotic technologies and information systems can also help reduce pre-analytical errors. Computerized order entry simplifies test ordering and eliminates a second person from transcribing the orders. In the post analytic phase there is the possibility of inappropriate response to the receipt, interpretation and utilization of laboratory information (5). It has recently been demonstrated that the introduction of new technologic facilities (online connection between laboratory and wards) without proper organization can worsen, rather than improve the communications between laboratories and clinicians (6).

At present major laboratories are using laboratory information systems (LIS) for reporting results solely or as part of hospital information system (HIS). HIS is comprehensive software for patient's information integration for sending and exchange comprehensive patient's information between wards and other medical centers in order to expedite the process of patient care, improve quality, increase satisfaction and reduce costs (7). Goal of HIS is using of computers and communications equipment for collecting, storing, processing, readout, and communication between patients cares with administrative data on all hospital activities and comply needs of all consumers system (8). In academic hospitals, also research and training support is one of the HIS goals; whereas laboratory information system (LIS) is part of HIS or it can function separately without HIS. 
The LIS is a data processing and dissemination technique used in the laboratory to deliver accurate and understandable results within a reasonable timescale as requested by clinicians. The system entails a sequence of events which include, delivery of samples to the laboratory, sample accessioning, analyzing, verifying and approving results or reanalyzing samples and releasing results to the clinicians who requested the tests (9). In short, the concept of LIS refers to the computerization of the laboratory system or automation of clerical laborintensive activities associated with the processing of laboratory results to improve accuracy and turnaround time of results (10).

\section{Materials And Methods}

Our hospital is a tertiary care government setup with obstetrics \& gynecology and pediatric services where the biochemistry lab runs majorly on manual procedures and semi automation with hand written reporting; and recently SINCE A YEAR, we are reporting by computers using online software \{Health4All, a Free and Open Source application supported by YouSee $\}$ as an upgradation of services in our hospital, but, without interfacing with the instruments. This software is a variant of LIS because test ordering has to be done at the laboratory level (Figure 1).

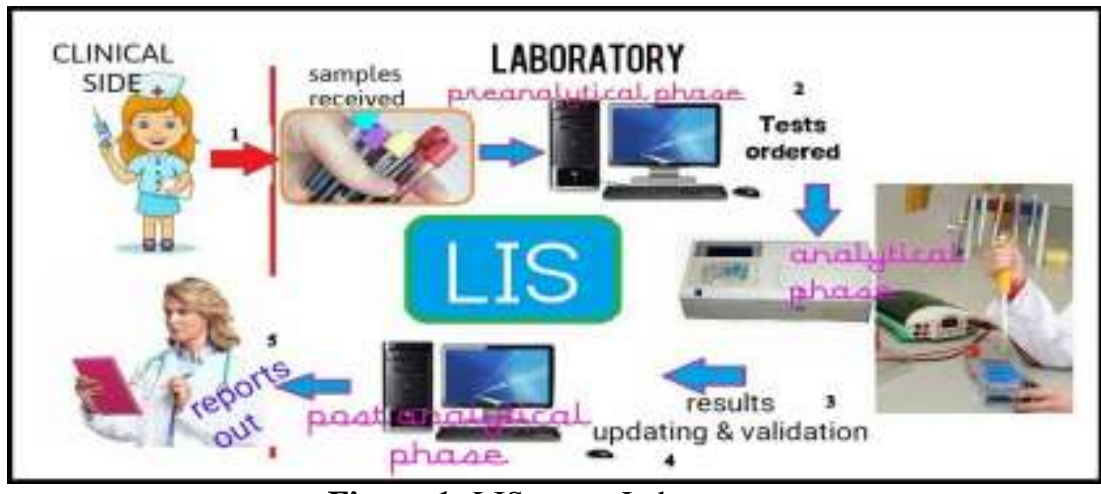

Figure 1: LIS at our Laboratory

With this background we planned this study to see the functionality, benefits and drawbacks of LIS in our hospital as a feedback to improve the LIS for better services to the patients.

We took the feedback by preparing a questionnaire and interviewing the clinicians $(\mathrm{n}=30)$, our laboratory staff $(n=15)$, nurses $(n=30)$ and the computer operators $(n=30)$, patient attendants $(n=30)$ to know regarding the functioning, advantages disadvantages of the online reporting and cross checked with the software programmers. The questions were prepared to answer as yes/no after explaining the purpose of the study. We also discussed a few important problems and drawn the conclusions.

\section{Resutls}

The format of questionnaire and the opinions were presented here. More than two thirds was taken as "majority". The questionnaire and the results were shown in Table 1.

\begin{tabular}{|c|c|c|c|}
\hline S.NO & QUESTION & $\begin{array}{l}\text { majority } \\
\text { YES }\end{array}$ & $\begin{array}{l}\text { majority } \\
\text { NO }\end{array}$ \\
\hline \multicolumn{4}{|c|}{ To the laboratory } \\
\hline 1. & Is it adding benefit to the lab in terms of time? & & $\sqrt{ }$ \\
\hline 2. & Is the work in the laboratory is more streamlined after LIS? & $\sqrt{ }$ & \\
\hline 3. & Does it decrease the manpower usage? & & $\sqrt{ }$ \\
\hline 4. & $\begin{array}{l}\text { Does it add any benefit in decreasing pre analytical errors like wrong } \\
\text { orders etc? }\end{array}$ & $\sqrt{ }$ & \\
\hline 5. & Does it streamline the reporting of emergency results? & & $\sqrt{ }$ \\
\hline 6. & $\begin{array}{l}\text { Does this eliminate the scribbling errors of manual reporting (post } \\
\text { analytical)? }\end{array}$ & & $\sqrt{ }$ \\
\hline \multicolumn{4}{|c|}{ To the Clinical staff } \\
\hline 1. & Does the result format which is used is informative? & & $\sqrt{ }$ \\
\hline 2. & Does it decrease the turnaround time compared to manual reporting? & & $\sqrt{ }$ \\
\hline
\end{tabular}


Effectiveness Of Online Reporting In A Laboratory Of A Tertiary Care Hospital - A Preliminary..

\begin{tabular}{|l|l|l|l|}
\hline & \multicolumn{1}{|c|}{} & & \\
\hline 3. & Can you directly access the results? & & $\sqrt{ }$ \\
\hline 4. & $\begin{array}{l}\text { Does the errors in reporting results decreased compared to manual } \\
\text { reports? }\end{array}$ & $\sqrt{ }$ & \\
\hline 5. & $\begin{array}{l}\text { Does it add any clinical benefit to the patient by giving accurate } \\
\text { analytical information? }\end{array}$ & $\sqrt{ }$ & \\
\hline
\end{tabular}

\begin{tabular}{|l|l|l|l|}
\hline \multicolumn{5}{|c|}{ To the patient attendants } \\
\hline 1. & Are you aware that the blood test reports come by computer? & $\sqrt{ }$ & \\
\hline 2. & Does this help in a better patient management? & $\sqrt{ }$ & \\
\hline 3. & Suggestions any? & $\sqrt{ }$ & \\
\hline
\end{tabular}

Apart from the questionnaire, we put forth a few problems which cannot be answered as yes or no; these problems were also discussed to draw solutions or suggestions. There are pros and cons;

The benefits of online reporting are:

1. It reduced the hassle at the laboratory reception area for test orders.

2. Decreased work burden to the laboratory technicians in the form of report writing and avoiding manual dispatch to the wards as well as duplicate reports.

3. Adds benefit to the patient as all the lab reports can be collected at one place (at ward's computer room) allowing quicker and better services.

4. A record of evidence of time and date of test order and reporting.

5. Storage QC (quality control) data and allows review for detection of laboratory errors.

6. Data is secure by password protection and data validation, approvals save time by working on two computers in parallel.

\section{The cons are:}

1. It is adding extra burden to the laboratory in terms maintenance of space and budget as well as computers (virus free scanning, power back up, network speed, wifi etc).

2. Storage of data or proper backup of results must be maintained as achieves for hospital statistical/research/surveillance purpose.

3. Recruiting more staff for computer rooms so that there would be no burden or pressure over existing man power in the wards or labs. Also computer staff must work round the clock in shifts, and to work in coordination with the lab personnel.

4. Proper training at level of wards (nurses, doctors) familiarizing the software as well as 'access'.

From this survey we found mixed results; this means there are, both, benefits as well as problems in reporting results online. Main suggestions received from the patients/attenders were, reports should be directly available at a kiosk type of machines installed at various places around the hospital premises. Many doctors and nurses wished to have a mobile access to those lab reports and out of range (high or low values) must be flagged to alert them.

\section{Discussion}

This study is a pilot study of on one year old LIS performance in serving patients for better care from the laboratory side. For this review we have done a thorough literature search to understand HIS, LIS and for similar studies but very few such studies were found. Tagger suggests that the functionality and usefulness of LIS mainly depends on the selection of the software which is proper for the hospital; if there are flaws in the selection process this will lead decreased productivity (11). After selection process, the functionality depends on the training of staff and how the services are utilized at the clinical end. From our results it is clear that improper training will create more problems rather adding benefit by LIS. Sepulveda and Young mentioned the characteristics of an ideal LIS; any software should have those characters to improve the result reporting (12). Our review has shown that if the selected software is having lacunae in design of test orders format and at data validation level, it would result in compromising the efficiency of LIS; therefore one need to go for revision/ upgradation of its features.

One of the advantages of the LIS is error detection thus improving the quality of a test result. Wang \& Ho conducted study on LIS for detection of errors in a laboratory, with and without interfacing instruments with LIS. It showed that interfacing improved the error detection (13). Though we work with semi-auto analyzers where interfacing with LIS computers is not possible, we found there was decreased error rate when compared back to manual reporting. 
Data from LIS also can be used to understand the volume of tests done, the time it takes to get results back to the patient and to plan efficient service delivery. It helps in improving the quality of laboratory results by participating in external quality programs by directly uploading the results and storing the quality results for further reference. In the testing process areas involving non-laboratory personnel, interdepartmental communication and cooperation are crucial to avoid errors. Therefore the entire health care system must be involved in improving the total testing process. But there must be adequate and effective training of personnel throughout the institution to be competent in following processes and procedures (14).

Therefore, understanding the challenges of the system and suggesting ways of improving the overall system performance would be a step in the right direction for a well-established and functioning laboratory to enhance quality and services.

\section{Conclusion}

The LIS, if carefully selected by the design and with proper training to use it, will work to simplify and improve the efficiency of laboratory operations, minimize data entry and other transcription errors as well as deliver valid and reliable laboratory results to the clinicians for swift and effective management of patients. And by regular review of LIS efficacy, its function has been used for appropriate and accurate review of all tests done in the laboratory and serves as platform for surveillance such as monitoring, utilization of retrospective data archived.

\section{Acknowledgements}

The authors would like to acknowledge HOD biochemistry Prof. Dr Vani Nutakki and Medical superintendent of Niloufer Hospital Dr C Suresh Kumar for their suggestions; And computer maintenance incharge Mr.Ranjith Bangari and software persons Mr. Shiva \& Mr.Gunaranjan P S (YouSee).

\section{REFERENCES}

[1]. Lippi G, Dimundic AM, Mattiuzzi C. Overview on patient safety in healthcare and laboratory diagnostics. Biochemia Medica. 2010; 20:131-143.

[2]. Mario Plebani, The detection and prevention of errors in laboratory medicine, Ann Clin Biochem 2010; 47: 101-110.

[3]. Lippi G, Mattiuzzi C, Plebani M. Event reporting in laboratory medicine. Is there something we are missing? MLO Med Lab Obs. 2009; 41:23.

[4]. Lippi G, Plebani M. The importance of incident reporting in laboratory diagnostics. Scand J Clin Lab Invest. 2009; 69:811-813. Boone DJ. Is it safe to have a laboratory test. Accred Qual Assur 2004; 10: 5-9.

[5]. Kilpatrick ES, Holding S. Use of computer terminals on wards to access emergency test results: a retrospective audit. BMJ 2001; 322:1101-3.

[6]. M. Aghajani, "Analytical and comparative study of hospital information systems," Medicine and Islam, 10th year, 2002; 47:36-29.

[7]. S. Aghazadeh, A. Aliyev, and M. Ebrahimnezhad, Review the Role of Hospital Information Systems in Medical Services Development, International Journal of Computer Theory and Engineering, 2012; 4: 866-870.

[8]. Paszko, C. (2014) Computerised Laboratory Information Management System (LIMS).

[9]. Peter S. Nyasulu, Christine Paszko, Nontombi Mbelle, a Narrative Review of the Laboratory Information System and Its Role in Antimicrobial Resistance Surveillance in South Africa, Advances in Microbiology, 2014; 4: 692-696

[10]. Tagger B. An introduction and guide to successfully implementing a LIMS (laboratory information management system). Aberystwyth: University of Wales. Available from:http://www.cs.ucl.ac.uk/staff/B.Tagger/LimsPaper.pdf

[11]. Jorge L. Sepulveda, MD, PhD; Donald S. Young, MD, PhD, The Ideal Laboratory Information System, Arch Pathol Lab Med. 2013;137:1129-1140.

[12]. Sihe Wang, PhD; Virginia Ho, BS, ASCP, Corrections of Clinical Chemistry Test Results in a Laboratory Information System, Arch Pathol Lab Med. 2004; 128: 890-892.

[13]. Da Rin G. Pre-analytical workstations: A tool for reducing laboratory errors. Clin Chim Acta. 2009; 404:68-74. 